\title{
An Effective SALS Image Denoising Method based on 2-D Discrete Wavelet Decomposition
}

\author{
Guang-ming Xian \\ Information Engineering and Technology Department, \\ South China Normal University,Foshan, Guangdong \\ 528225, China \\ E-mail:xgm20011@126.com
}

\author{
Bi-qing Zeng \\ Information Engineering and Technology Department, \\ South China Normal University,Foshan, Guangdong \\ 528225, China
}

\begin{abstract}
This paper describes an effective small angle light scattering (SALS) image denoising method based on 2-D discrete wavelet decomposition. The phenomenon of small angle light scattering has been applied to giving information about transparent structures on morphology. The potential of using SALS to probe morphological changes induced by flow in polymer melts is investigated. Well-defined flow of polymer melts histories in a visual slit die are shown to result in SALS patterns that are characteristic for the morphology involved. The current study utilizes a 2-D discrete wavelet to delete noise from the original SALS image and estimate the variation trend of maximum intensity area of the scattered light. To compare the SALS denoising result, four commonly used wavelets, named haar wavelet, db2 wavelet, Coi4 and Sym5 wavelet are taken into account. It can also be observed that $\mathrm{db} 2$ wavelet obtain the best denoising result in the four wavelts.
\end{abstract}

Keywords- Small angle light scattering image; 2-D discrete wavelet decomposition; polymer characterization; image denoising.

\section{Introduction}

Light scattering techniques which give information on optical properties have been proven to be useful for obtaining deep insights into the molecular and structural parameters of polymers. These techniques are especially useful for polymeric systems with supermolecular structures in solutions, liquids and solids. The main advantage is that the measurement does not damage or interrupt the system [1].

Small angle light scattering (SALS) techniques offer a number of advantages for the investigation of the nature and behavior of polymer materials. Non-intrusive characterization of the flow field of transparent film is an essential step towards an implementation of a structural control system that can regulate the structure development during processing.

Understanding the connection between applied flow fields and morphology is vital to the intelligent processing and control of polymer melts properties. The development of techniques capable of providing in situ morphological information is a key step in this understanding [2]. The purpose of this work is to demonstrate that small angle light scattering, is a viable technique for following changes in the morphology of polymer melts.

The properties of polymer blends are determined to a large extent by the morphology that is developed during the processing. As the industrial applications of polymer blends increase [3], there is an increasing need for developing a better understanding of the relationships between melt deformation history, phase behavior and morphology development. Knowledge of these relationships is necessary for understanding the origin of the morphology produced in commercial blends processes, and it also may provide the opportunity for attaining better control of the blend morphology during processing and, perhaps, achieving unique structures and properties [4].

Typical polymer processing procedures, e.g. extrusion or injection molding, subject the polymer blend to complicated deformations under non-isothermal conditions. The deformation field is typically heterogeneous and includes both shear and elongational strains, and it is not conducive to easy analysis, especially if one wants to know the phase behavior of a blend under stress and achieve a cause-and-effect understanding of how structure develops. Studies involving well-controlled deformations, e.g. simple shear, are much easier to accomplish, and although such deformations oversimplify an actual melt processing operation, they do provide a basis for developing an understanding of the more complicated flows [4].

Recently the problem of SALS image denoising has received attention. We propose that 2-D wavelet decompositon algorithms can be used to perform SALS image denoising. Consequently, a variety of new image denoising techniques becomes available. Fei Shi, Selesnick, I.W., and Shihua Cai examine the sharpening of natural images using an algorithm for image denoising with oriented complex 2D wavelets [5]. The method of wavelet thresholding based on image denoising is presented in paper [6]. An image contaminated by white noise is decomposed in a separable two-dimensional wavelet basis. A wavelet thresholding algorithm which is well adapted to suppressing noise from a non-textured image is introduced to produce a better image quality with a higher SNR. Chaux, C., Duval L., Benazza-Benyahia A., and Pesquet J.-C. [7] propose a new estimator for image denoising using a 2D dual-tree $\mathrm{M}$ band wavelet transform. Our work extends existing blockbased wavelet thresholding methods by exploiting simultaneously coefficients in the two M-band wavelet trees. The contributions of this paper are two-fold. Firstly, we perform a statistical analysis of the noise in the considered redundant decomposition. Secondly, we propose an efficient 
method to remove the noise. Their approach relies on an extension of Stein's formula which allows us to take into account the specific correlations of the noise components. Simulation results are then presented to validate the proposed method.

Wavelet analysis ( WA ) is typically suited in applications where data contains both large and small scales of variation, such as small angle light image. We presented a new technique that can be used to analysis the structural information of transparent film on- line and non-intrusively while the material is processing. The technique is based on SALS, optical signal real-time analysis software and wavelet transform method. It is shown that the proposed technique is easy to implement and provides more flexibility approximating the relation between the intensity image and the corresponding variation time. Applying this method to analyze structural information of transparent film will be of great interest, since it will contribute information on optical prosperities have been proven to be useful for obtaining deep insights into the molecular and structural parameters of transparent film. In our experiments, the SALS image is de-noised by 2-D wavelet decomposition. The variation trend of the SALS image becomes clear and the exceptional SALS image can be accurately detected by wavelet decomposition [8].

In this paper, the results from a measurement technique are investigated. The method will be evaluated on the basis of light scattering measurements for a small range of scattering angles. These measurements have been taken with a fast CCD line scan camera and appropriate optics. An attempt is made to derive information from these measurements only. The purpose of the present work is to apply optical image technique to characterization the structural informal of transparent film. 2-D discrete wavelet decomposition technique is used to denoise the small angle light scattering image.

\section{THEORETICAL BACKGROUND FOR SALS}

When a light beam passes through a diffusion surface, the variation of propagation direction of the beam can not be determined by the principle of geometrical optics because of scattering function of light beams on diffusion surface [9].

A transparent fluid is an optical phase object. In the experimental set-up for measuring flow fields in fluid flows by using speckle interferometry, the part of the arrangement for the object light beam is just like a subjective photographic system. Therefore, in general, speckle displacements are generated. The speckle displacements can change the intensity distribution of spatial speckle fields. As a result, the intensity distribution of a speckle interferogram is also changed. In this paper the effect of variation of the intensity is analyzed and discussed in detail. Experimental results are shown. Methods for elimination of the multiple scattering effect are provided. This is advantageous to improve the quality of the speckle interferogram [10].

In our experiments, device performs real-time image analysis of the evolving light scattering signal. The experimental device incorporates a $\mathrm{He}-\mathrm{Ne}$ laser generator, optics, a CCD camera, and a personal computer as its major hardware components. Software designed specifically for this application performs real time analysis of the light scattering pattern. Intensities at various scattering and azimuthal angles are plotted at each time [11].

A laser light passes through polymer melts in the visual slit die. A polarizer and an analyzer are placed before and after the polymer melts. The laser light first passes the polarizer, which removes one orthogonal component of the light [12]. The other component of light passes through the polymer melts with resulting scattering due to the orientation of molecular chain. The analyzer removes the second component since it is placed out of phase with respect to the analyzer. Therefore, any light that comes out of the analyzer is entirely due to the scattering within the polymer melts. The depolarized intensity of light that passes through the polarizer, polymer melts and analyzer is recorded and related to the orientation of polymer melts. A CCD camera captures the image and the total intensity of the image is determined in every $5 \mathrm{~s}$.

According to the effect of the sample on the incident light, the sample can be divided into a surface layer, a first scattering layer, a second scattering layer, etc. The incident light first impinges on the surface of the sample the first scattering layer (random reflection) of the medium. The second one comes from the first scattering layer (because of the internal heterogeneity) and third in turn [1].

It is assumed that there is no energy loss accompanying the scattering and that the scattering medium is sphere symmetric. Debye and Bueche 2 applied statistical theory to heterogeneous solid scattering and proposed that, in the range of small scattering angles, the scattering light is described by

$$
I_{(h)}=K^{\prime} \int_{0}^{\infty} \gamma_{(r)} \frac{\sin (h r)}{h r} r^{2} d r
$$

when $\gamma_{(r)}$ is a correlation function corresponding to fluctuation of the medium. Generally, for polymers:

$$
\gamma_{(r)}=\exp \left(-r / a_{c}\right)
$$

is the correlation distance and $h=(4 \pi / \lambda) \sin (\theta / 2))$ is a scattering vector. Substituting equation (4) into equation (3) and integrating, we can obtain correlation distance as follows:

$$
\frac{1}{\left[I_{(h)}\right]^{1 / 2}}=\frac{1}{\left(k^{\prime \prime} a_{c}^{1 / 2}\right)^{3}}\left(1+h^{2} a_{c}^{2}\right)
$$

When $r=0, \gamma_{(r)}=1$, we obtain integral area :

$$
\begin{aligned}
& \overline{\eta^{2}}=K^{\prime} \int_{0}^{\infty} I_{(h)} h^{2} d h \\
& Q=\int_{0}^{\infty} I_{(h)} h^{2} d h
\end{aligned}
$$


The parameter describes the distance the heterogeneity expands in the scattering medium, and is the intensity of heterogeneity of the scattering object [1].

\section{2-D WAVELET DECOMPOSITION FOR SALS IMAGE}

The mathematics of the wavelet transform were extensively studied and can be referred in papers [13] and [14]. The multiresolution analysis was introduced by Mallat and a detailed study about MRA can be found in [15].

Basically, a wavelet is a function with a zero average

$$
\int_{-\infty}^{+\infty} \psi(t) d t=0
$$

The continuous wavelet transform (CWT) of a signal is then defined as

$$
C W T_{\psi} x(a, b)=\frac{1}{\sqrt{|a|}} \int_{-\infty}^{+\infty} x(t) \psi^{*}\left(\frac{t-b}{a}\right) d t
$$

where $\psi(t)$ is called the mother wavelet, the asterisk denotes complex conjugate, $a$ and $b(a, b \in R)$ are scaling (dilation) and translation parameters, respectively. The scale parameter $a$ will decide the oscillatory frequency and the length of the wavelet, the translation parameter $b$ will decide its shifting position.

In a practical application, we will use the discrete wavelet transform (DWT) instead of the CWT. This is implemented by using discrete values of the scaling parameter $a$ and $b$ translation parameter $b$. To do so, set $a=a_{0}^{m}$ and $b=n b_{0} a_{0}^{m}$, then we get

$$
\psi_{m, n}(t)=a_{0}^{-m / 2} \psi\left(a_{0}^{-m} t-n b_{0}\right)
$$

where $m, n \in Z$ and $m$ indicating frequency localization and $n$ indicating time localization. Generally, we can choose $a_{0}=2$ and $b_{0}=1$. This choice will provide a dyadic-orthonormal wavelet transform and provide the basis for multiresolution analysis.

InMRA, any time series $x(t)$ can be completely decomposed in terms of the approximations, provided by scaling functions $\phi_{m}(t)$ and the details, provided by the wavelets $\psi_{m}(t)$, where $\phi_{m, n}(t)$ and $\psi_{m, n}(t)$ are defined as the following:

$$
\begin{aligned}
& \phi_{m, n}(t)=2^{-m / 2} \phi\left(2^{-m} t-n\right) \\
& \psi_{m, n}(t)=2^{-m / 2} \psi\left(2^{-m} t-n\right)
\end{aligned}
$$

(8)

The scaling function is associated with the low-pass filters with filter coefficients $\{h(n), n \in Z\}$, and the wavelet function is associated with the high-pass filters with filter coefficients $\{g(n), n \in Z\}$. The so-called Two Scale Equations (TSE) give rise to these filters.

$$
\begin{aligned}
& \phi(t)=\sum_{n} h(n) \sqrt{2} \phi(2 t-n) \\
& \psi(t)=\sum_{n} g(n) \sqrt{2} \phi(2 t-n)
\end{aligned}
$$

There are some important properties of these filters

$$
\begin{array}{llll}
\sum_{n} h(n)^{2}=1 & \text { and } & \sum_{n} g(n)^{2}=1 \\
(7) & \text { and } & & \sum_{n} g(n)=0
\end{array}
$$

Filter $g(n)$ is an alternating flip of the filter $h(n)$, which means there is an odd integer $N$ such that

$$
g(n)=(-1)^{n} h(N-n)
$$

\section{EXPERIMENTAL RSULTS}

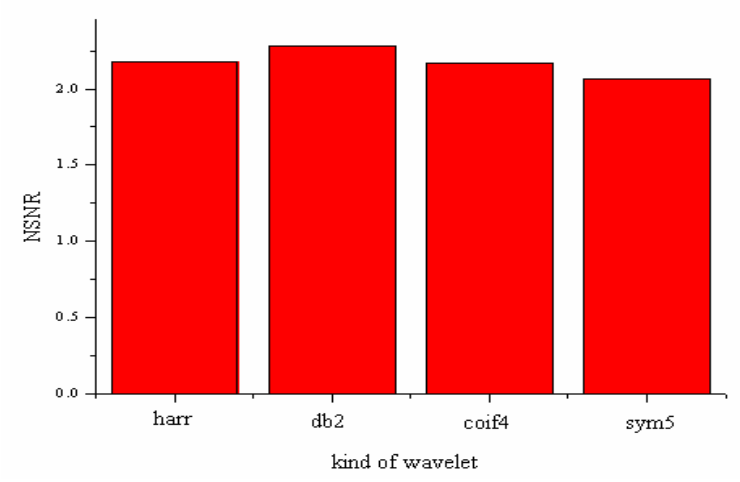

Fig.1 The values of NSNR under different noise levels

The 2-D DWT of a 2-D signal can be implemented by using the 1-D DWT in a separable fashion. At each level of decomposition (or reconstruction), the 1-D forward DWT (or inverse DWT) is first applied to every column of the resulting data.

An import issue related to the proposed DWT method is the choice of a suitable wavelet. To compare the SALS denoising result, four commonly used wavelets, named haar wavelet, db2 wavelet, Coi4 and Sym5 wavelet are taken into account. 
The values of NSNR under differ rent kind of wavelet were listed in Fig. 1. It can also be observed that $\mathrm{db} 2$ wavelet obtain the best denoising result in the four wavelts.

Denoising results of SALS image obtained by DWT method were shown in Fig. 2. It is easily to see that the noise has been effectively reduced and the visual effect has been highly enhanced compared with the corresponding noise versions.

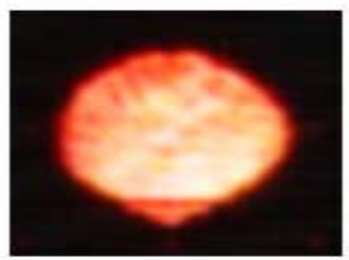

(a)

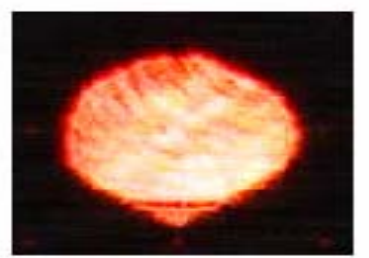

(b)
Fig.2 Original image and denoising image (a) image with noise (b) denoised image

We choose Db2 wavelet and scan the decomposition levels from 1 to 8. As we can see from Fig. 3, When the decomposition level is equal to 5 , we can achieve the best SALS image deniosing result.

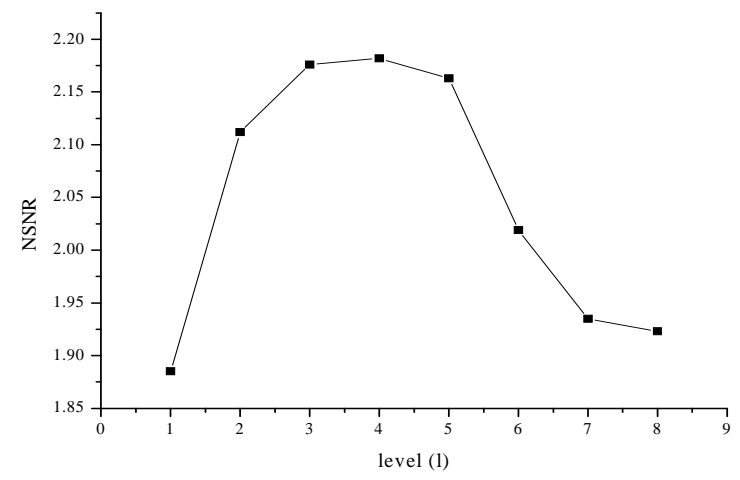

Fig.3 Deniosing result of scan the decomposition levels from 1 to 8 by using db2 wavelet

\section{CONCLUSIONS}

The present work results in an effective technique for denoising SALS image based on discrete wavelet decomposition. The analysis of specific features of the SALS pattern provides an accurate technique for extracting information from these patterns which can characterize the flow field of polymer melts. 2-D DWT was performed to get denoised SALS image. The current study utilizes a 2-D discrete wavelet to delete noise from the original SALS image and estimate the variation trend of maximum intensity area of the scattered light. So the system brought the qualitative analysis of the structural information of transparent film success. We choose Db2 wavelet and scan the decomposition levels from 1 to 8. When the decomposition level is equal to 5, we can achieve the best SALS image deniosing result.

\section{ACKNOWLEDGEMENT}

The authors acknowledge the support of the South China Normal University and South China University of Technology. The work was support by the project of research of support vector machine in classification and regression, under project number Guangdong financial education (2008) 342. The work was also support by Guangdong province natural science fund (project No. 8151063101000040).

\section{REFERENCES}

[1] Jiamin Zhou and Jing Sheng. Small angle light backscattering of polymer blends: 1 . Multiple scattering. Polymer Vol. 38 No. 15, pp. 3727-3731, 1997

[2] J. Mewis, H. Yang, P. Van Puyvelde, P. Moldenaers and L. M. Walker. Small-angle light scattering study of droplet break-up in emulsions and polymer blends. Chemical Engineering Science, Vol. 53, No. 12, pp. 2231-2239, 1998

[3] Utracki LA. Polymer alloys and blends: thermodynamics and rheology, New York: Oxford University Press, 1989.

[4] Z. Hong, M.T. Shawa, R.A. Weiss. Structure evolution and phase behavior of polymer blends under the influence of shear. Polymer 41 (2000) 5895-5902.

[5] Fei Shi, Selesnick, I.W., Shihua Cai. Image sharpening via image denoising in the complex wavelet domain. Proceedings of the SPIE The International Society for Optical Engineering, v 5207, n 1, 14 Nov. 2003, 467-74

[6] Keita Alpha, Peng Jiaxiong. Wavelet thresholding based on image denoising. Journal of Huazhong University of Science and Technology, v 29, n 6, June 2001, 13-15

[7] Chaux, C., Duval L., Benazza-Benyahia A., and Pesquet J.-C.. A new estimator for image denoising using a 2D dual-tree M-band wavelet decomposition. 2006 IEEE International Conference on Acoustics, Speech, and Signal Processing (IEEE Cat. No. 06CH37812C), 2006, III249-52.

[8] Xian Guangming; Wang, Zhiyan. An effective technique of wavelet transform for optical signal real-time processing. 2005 International Conference on Communications, Circuits and Systems - Proceedings, 2005, v 1, p 653-657

[9] Wayne T. Gulberson, and Martin R. Tant, "Device for study of polymer crystallization kinetics via real-time image analysis of small angle light scattering,” J. Appl. Polym. Sci., vol.47, 1993, pp.395-405.

[10] Yaozu Song, and Wei Zhang, "Laws of laser speckle movement and their application in flow visualization,” in Proc. SPIE. Int. Soc. Opt. Eng., vol. 3783, 1999, pp.89-100.

[11] Yaozu Song, R. Kulenovic, M. Groll, and Zengyuan Guo, "Effects of speckle displacement on speckle interferometry for measurement of phase object,” Optics Communications, vol.139, pp. 24-30, June 15, 1997.

[12] Batur Celal, Hanif Vhora Mohamad, Cakmak Milo, and Serhatkulu Toprak, "On-line crystallinity measurement using laser Raman spectrometer and neural network,” ISA Transactions 38, 1999, pp.139148.

[13] I. Daubechies, “Ten lectures on wavelets,”. Philadelphia, PA: Society for Industrial and Applied Mathematics, 1992.

[14] C. S. Burrus, R. A. Gopinath, and H. Guo, Introduction to Wavelets and Wavelet Transforms: A Primer. Englewood Cliffs, NJ: Prentice-Hall, 1998.

[15] S. Mallat, "A theory for multiresolution signal decomposition: The wavelet representation,” IEEE Trans. Pattern Anal. Mach. Intell., vol. 11, no. 7, pp. 674-693, Jul. 1989. 